\title{
Commentary on 'A longitudinal qualitative evaluation of patient perspectives of adverse events after pelvic reconstruction surgery'
}

\author{
Abdelmageed Abdelrahman ${ }^{1}$
}

Received: 18 June 2019 / Accepted: 17 July 2019/Published online: 13 August 2019

(C) The International Urogynecological Association 2019

This was a mixed-method study of longitudinal patient interviews and surveys conducted at the University of New Mexico. The primary aim of the study was to longitudinally examine how patients view surgical adverse events over time and explore how such perspectives may change from the preoperative period to 6 months after surgery.

Each study participant had three interviews (preoperatively, immediately postoperatively and late postoperatively) by the same trained research coordinator. At the preoperative interview, participants completed the Pelvic Floor Distress Inventory Questionnaire-Short Form 20 (PFDI 20) and the Life Orientation Test-Revised (an assessment of optimism). At the 6-8-week and 6-month postoperative interviews, participants completed the PFDI 20, the Patient Global Impression of Improvement (PGI-I), the Satisfaction with Decision Scale-Pelvic Floor Disorders (SDS-PFD) and the Decision Regret Scale-Pelvic Floor Disorders (DRS-PFD).

The study period was October 2017 to January 2018 with 20 women who had surgery recruited. Participants underwent a variety of surgeries including mid-urethral slings, sacral neuromodulation, vaginal native tissue repairs and laparoscopic hysterectomy. It was noted that women's perceptions of adverse events changed as more time passed from surgery. Preoperatively, women expressed concerns about anaesthesia complications, pain, injury, catheter issues and unsuccessful surgery. At 6-8 weeks postoperatively women identified concerns regarding functional outcomes. At 6 months postoperatively the majority of women identified unsuccessful surgery, incontinence and sexual dysfunction as severe adverse events.

Strengths of this interesting study include the use of a robust qualitative methodology with validated questionnaires and skilled qualitative researchers. Another important strength was the ability to complete three rounds of interviews with all studied patients. Limitations of the study include the small sample size of 20 participants.

\section{Compliance with ethical standards}

Conflicts of interest None.

Publisher's note Springer Nature remains neutral with regard to jurisdictional claims in published maps and institutional affiliations.

Abdelmageed Abdelrahman

abdelmageed@hotmail.co.uk

1 Department of Urogynaecology, Liverpool Women's Hospital NHS Foundation Trust, Liverpool, UK 